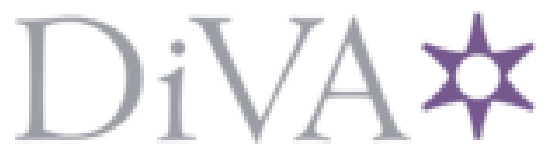

http://www.diva-portal.org

This is the published version of a paper presented at 13th International Conference on Modern Problems of Radio Engineering. Telecommunications and Computer Science (TCSET).

Citation for the original published paper:

Kuzminykh, I. (2016)

Development of traffic light control algorithm in smart municipal network In: IEEE (ed.), 13th International Conference on Modern Problems of Radio Engineering. Telecommunications and Computer Science (TCSET), 23-26 Feb. 2016, Lviv, Ukraine Lviv, Ukraine: IEEE https://doi.org/10.1109/TCSET.2016.7452218

N.B. When citing this work, cite the original published paper.

Permanent link to this version:

http://urn.kb.se/resolve?urn=urn:nbn:se:bth-15953 


\title{
Development of Traffic Light Control Algorithm in Smart Municipal Network
}

\author{
Ievgeniia Kuzminykh
}

\begin{abstract}
This paper presents smart system that bypasses the normal functioning algorithm of traffic lights, triggers a green light when the lights are red or reset the timer of the traffic lights when they are about to turn red. Different pieces of hardware like microcontroller units, transceivers, resistors, diodes, LEDs, a digital compass and accelerometer will be coupled together and programed to create unified complex intelligent system.
\end{abstract}

Keywords - Smart City, Smart Mobility, Microcontroller, STM32, Traffic Light.

\section{INTRODUCTION}

A smart city (also smarter city) uses digital technologies or information and communication technologies (ICT) to enhance quality and performance of urban services, to reduce costs of living and resource consumption and to engage more effectively and actively with its citizens. Sectors that have been developing smart city technology include government services, transport and traffic management, energy, health care, water and waste management services. Smart city applications are developed with the goal of improving the management of urban flows and allowing for real time responses to challenges [1].

A smart city is built upon the following elements:

- Smart Mobility

- Smart Living

- Smart People

- Smart Government

- Smart Economy

- Smart Environment

In this paper we focus on Smart Mobility. Mobility sector is broken down into 5 layers [2] as follows:

1) User Experience Layer, this is the layer whereby the user receives shipping, information, or other services from the transportation company, and where transportation actually occurs.

2) Transportation Services Layer, this is the layer whereby transportation companies provide services to users.

3) Information Collection Layer, at this layer the information is gathered regarding how the services provided by transportation companies is used by users.

4) Information Management and Control Layer, at this point the information is managed and controlled in order to ensure that transportation companies smoothly provide the services in the transportation services layer.

Ievgeniia Kuzminykh - Kharkov National Universit of Radio Electronic, Lenina Av. 14, Kharkov, 61166, UKRAINE, E-mail: yevheniia.kuzminykh@nure.ua
5) Transportation Company Coordination Layer, this is the point whereby each transportation company's information is gathered, analyzed, and provided, in order to optimize transportation inside the entire city, thereby guiding the operation and control of the transportation companies.

We shall be working within layer 4 of the smart mobility structure.

The main result should be to setup both hard and software which will be aimed at helping control traffic lights for high Priority vehicles such as ambulances, firefighting trucks, military and police cars, VIP and presidential convoys etc. at roundabouts and junctions to grant them swift passage needed to arrive at their destination and reduce on sirens noise around the cities.

\section{PROJECT DEVELOPMENT}

\section{A. Topology of project}

The operating principle of the system is as follows. As the vehicle carrying this intelligent system (ambulance, fire brigade, police and other emergency vehicles) approaches a certain junction/roundabout, based on the distance programmed in the vehicle inbuilt device to triggers the traffic lights, they are automatically switched to green in order to allow movement without stopping at the junction. High priority vehicles are always in a rush to get to their destination without being inconvenienced by traffic lights. 30 or $45 \mathrm{sec}$ can mean a lot for a life while it tries to maneuver through heavy traffic.Topology of developed system is shown in fig.1.

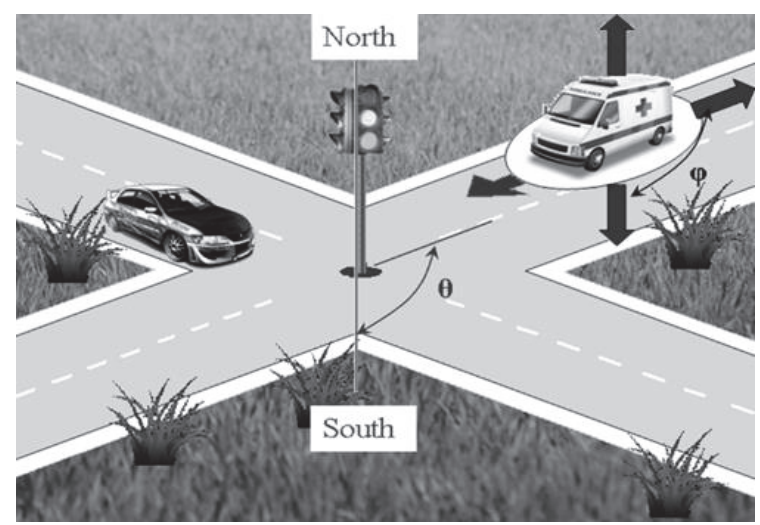

Fig.1. Topology of Developed System

The required direction of movement is automatically calculated using special equipment. The car is installed with a microprocessor system with an electronic compass with an embedded accelerometer and a transmitter of a limited range $(50 \ldots 100 \mathrm{~m})$. At the traffic 
lights a management system is installed with a transceiver radio module that processes the information from moving objects.

The traffic lights work with a known orientation, and the orientation and direction of movement of the vehicle movement are calculated by the on-board microprocessor system. This information is constantly processed and transmitted and once the car is approaching the traffic lights in the programmed range, the information is relayed to the traffic lights changing the normal functioning algorithm, hence switching the lights to the desired direction green.

\section{B. Hardware development}

Figure 2 shows a block diagram of a microprocessor unit and how different units are connected to it in order to achieve a smart system that overrides the normal function algorithm of traffic lights to grant any high priority vehicle clear passage on busy road Junctions or roundabouts. This is receiver side.

The setup consists of the following units:

- $\mathrm{MC}$ - microcontroller;

- Power supply;

- Voltage regulator;

- Debug port;

- Digital compass/ magnetometer;

- Transceiver;

- Traffic light.

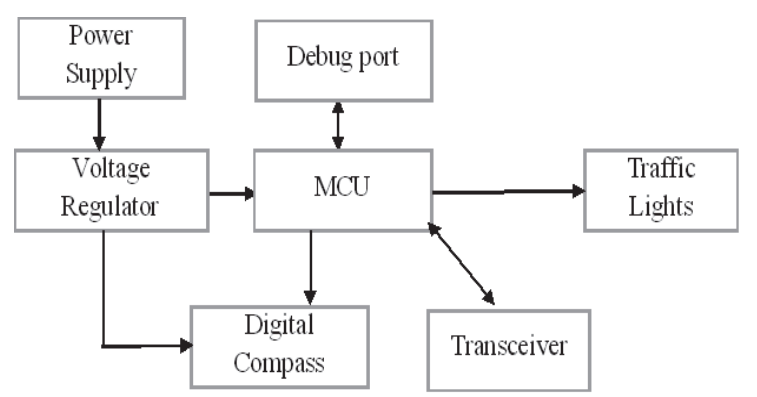

Fig.2. Block Diagram for Setup

The Microcontroller Unit (MCU) is used to control the entire device and coordinate between the different components of the system. In this project, we used stm32f050f4p6 microcontroller [3]. Its circuit design is displayed on fig.3.

This MCU is an Arm 32- bit Cortex- M0 CPU with an operating frequency of up to $48 \mathrm{MHz}$. Memories is from 16 to 32 Kbytes of Flash memory, 4 Kbytes of SRAM with HW parity checking, CRC calculation unit, Reset and supply management, Voltage range: $2.0 \mathrm{~V}$ to $3.6 \mathrm{~V}$, Low power modes: Sleep, Stop and Standby.

The tests were done using Nordic semiconductor's nRF24L01 [4] wireless transceiver with $2.4 \mathrm{GHz}$ ISM band operation and up to $2 \mathrm{Mbps}$ on air data rate. This transceiver unit was chosen due to its low cost of production, easy programing, and compatibility to the system, low operating temperature, size and power efficiency.

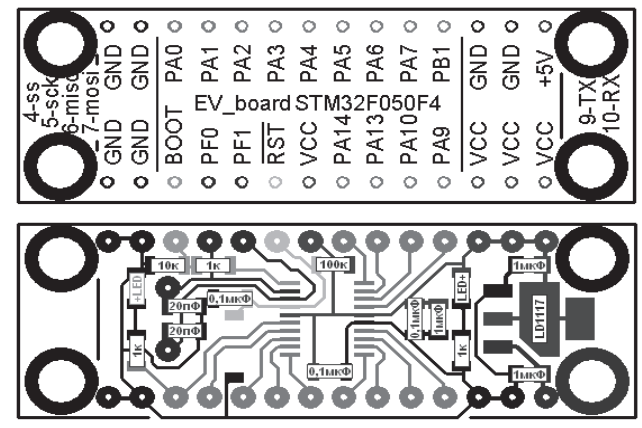

Fig.3. STM32f050f4p6 Microcontroller Circuit Design

Knowing the direction from which the signal is being generated is very important in this project. Since we are dealing with traffic and traffic lights, it would be dangerous or even catastrophic if all traffic lights were triggered to turn green, so the direction the transmission is coming in from is very critical and no better way to ensure accuracy than using a digital compass/ magnetometer.

For this test, we used the HMC5883L magnetometer.

HMC5883L [5] with the following benefits that made it the best choice for the project:

1) Small Size for Highly Integrated Products. Just Add a Microcontroller Interface, Plus Two External SMT Capacitors Designed for High Volume, Cost Sensitive OEM Designs Easy to Assemble \& Compatible with High Speed SMT Assembly;

2) Enables $1^{\circ}$ to $2^{\circ}$ Degree Compass Heading Accuracy;

3) Enables Low-Cost Functionality Test after Assembly in Production;

4) Compatible for Battery Powered Applications.

5) After assembling all components, the device is ready for programming (fig.4).

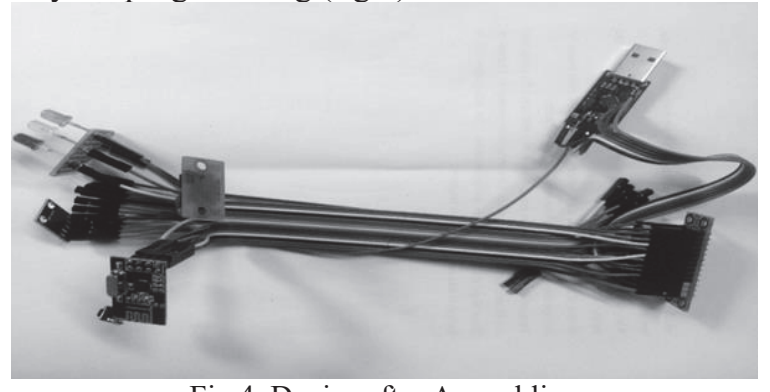

Fig.4. Device after Assembling

C. Software development and algorithm

Fig. 5 shows the traffic light control algorithm.

Fig. 6 shows the car operation algorithm. 


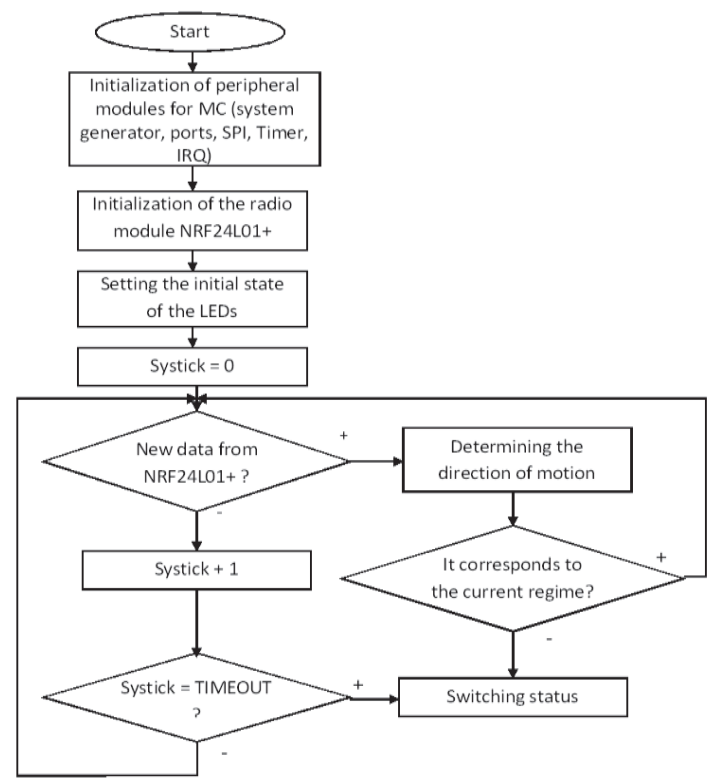

Fig.5. Smart Traffic Light Control Algorithm

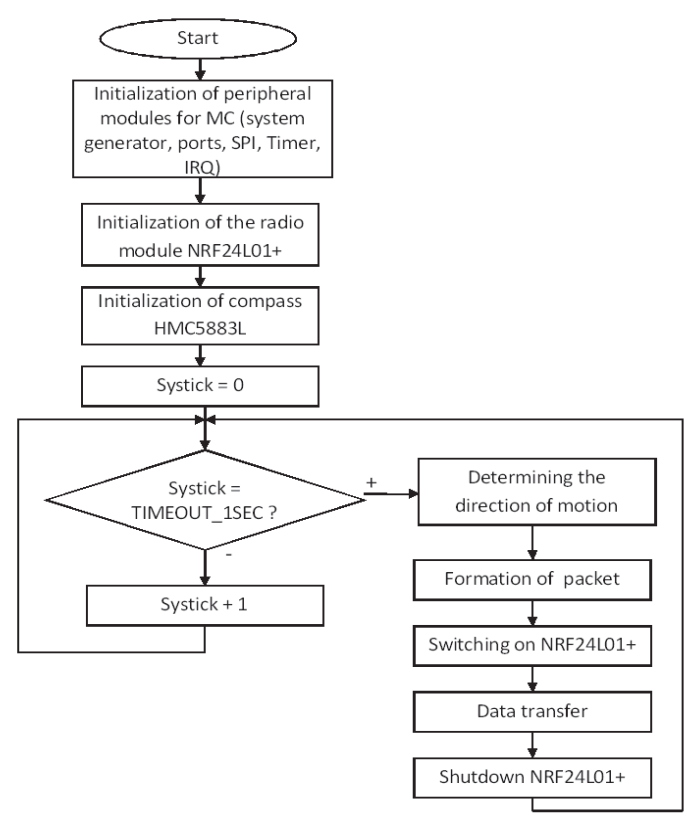

Fig.6. The Algorithm of Functioning of Mobile Systems

\section{CONCLUSION}

This work looks at the kind information and telecommunications needed to develop and nurture a smart city. It lists six essential elements for any smart city ICT planning policy. These six essentials are closely related and build upon each other to ultimately provide a comprehensive ICT solution. This solution takes the form of a ubiquitous digital platform, which is available across the city (via Wi-Fi hotspots, private networks or local information kiosks) to all members of the smart city or community. It facilitates collaboration at a local level in a streamlined digital fashion, reducing energy and time costs for all participants and increasing quality of life within the community.

In this paper we concentrated on smart mobility and our emphasis was on layer four of smart mobility which deals with information management and control. Our task is to create a system that bypasses the normal functioning algorithm of traffic lights, triggers a green light when the lights are red or to reset the timer of the traffic lights when they are about to turn red. Different pieces of hardware like microcontroller units, transceivers, resistors, diode, LEDs, digital compass and accelerometer were coupled together and programed to create one complex intelligent system that eased movement of high priority vehicles around smart cities.

\section{REFERENCES}

[1] N. Komninos, "The architecture of intelligent cities: Integrating human, collective and artificial intelligence to enhance knowledge and innovation," The 2nd IET International Conference on Intelligent Environments,vol. 1, pp. 13-20, 2006.

[2] T. Okuda, S. Hirasawa and et. "Smart Mobility for Smart Cities", Hitachi Review, Vol. 61, No. 3, pp.141-146, 2012.

[3] "STM32F050x4 STM32F050x6". Datasheet, STMicroelectronics group of companies, 97p., 2012.

[4] "nRF24L01+.Single chip $2.4 \mathrm{GHz}$ transceiver. Preliminary Product_Specification v1.0." Nordic Semiconductor, $75 \mathrm{p}$.

[5] "3-Axis Digital Compass IC HMC5883L", Honeywell's Magnetic Sensors' Advanced Information, 20 p., 2013. 